\title{
Activated $\beta$-catenin Forces N2A Cell-derived Neurons Back to Tumor-like Neuroblasts and Positively Correlates with a Risk for Human Neuroblas-
}

\section{toma}

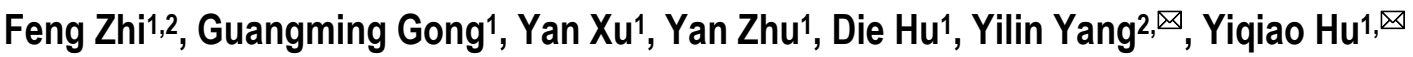 \\ 1. State Key Laboratories of Pharmaceutical Biotechnology, School of Life Sciences, Nanjing University, Nanjing, China \\ 2. Third Affiliated Hospital of Soochow University, Changzhou, China
}

$\bowtie$ Corresponding author: Yilin Yang, E-mail: yilinyang.czfph@gmail.com; and Yiqiao Hu, E-mail: huyiqiao.nju@gmail.com

( ) Ivyspring International Publisher. This is an open-access article distributed under the terms of the Creative Commons License (http://creativecommons.org/ licenses/by-nc-nd/3.0/). Reproduction is permitted for personal, noncommercial use, provided that the article is in whole, unmodified, and properly cited.

Received: 2011.09.15; Accepted: 2012.01.16; Published: 2012.01.23

\begin{abstract}
Neuroblastoma is an embryonic malignancy arising from neuroblasts. The mechanisms that regulate the origination of neuroblastoma are still not very clear. In this study, we revealed that 6-bromoindirubin 3'-oxime (BIO), a specific GSK-3 $\beta$ inhibitor, promoted N2A cells-derived neurons to become tumor-like neuroblasts. Moreover, constitutively activated $\beta$-catenin (S33Y) also promoted this process, whereas, silencing endogenous expression of $\beta$-catenin abolished $\mathrm{BIO}$-induced effects. These results implicated the potential relationship between the $\mathrm{Wnt} / \beta$-catenin signaling and neuroblastoma formation. Indeed, we found that the amount of $\beta$-catenin in nucleus, which indicated the activation of $\mathrm{Wnt} / \beta$-catnin signaling, was accumulated in human neuroblastoma specimens and positively correlated with clinical risk of neuroblastoma. These results give us a new sight into the neuroblastoma initiation and progression, and provide a potential drug target for neuroblastoma treatment.
\end{abstract}

Key words: Neuroblastoma, GSK-3 $\beta$, $\beta$-catenin, neuroblasts

\section{Introduction}

Neural development is a strictly controlled process that integrates proliferation, differentiation, and programmed cell death or apoptosis [1]. Abnormal neural development may lead to emotional, behavioral and learning problems in children, even the growth of neural tumors [2]. Neuroblastoma, an embryonic malignancy of the nervous system arising from neuroblasts [3], is the most common extracranial solid tumor of childhood. Although substantial progress has been made in identifying neuroblastoma specific molecular targets, it still remains a complex medical challenge to understand the molecular mechanism of neuroblastoma initiation, differentiation and regression. Recently, studies have focused on the molecular link between neural development and the genesis of neuroblastoma.
Wnt/ $\beta$-catenin signaling is correlated with numerous cancers, such as intestine, breast, prostate and lung cancers. Dysregulation of this pathway plays an important role in a number of adult and pediatric tumors, which indicates the important role of Wnt/ $\beta$-catenin signaling pathway in tumors [4]. In addition, canonical Wnt signaling through the $\beta$-catenin/TCF/LEF transcription complex has been demonstrated to play a central role in neural crest induction [5] and progenitor cell fate determination in both embryonic and adult stem cells [6-7]. It has been reported that $\mathrm{Wnt} / \beta$-catenin signaling could promote neural stem cell (NSC) proliferation [8] and prevent neuronal differentiation [9], even reprogram somatic cells into pluripotent cells [10-11]. Although 
Wnt $/ \beta$-catenin signaling plays essential roles in many tumors and is involved in neural development, its role in neuroblastoma has not been well explored.

In this study, we investigated the role of Wnt/ $\beta$-catenin in modulation of cellular plasticity of the N2A cells-derived neurons and its possible functions in origination of neuroblastoma. We found that 6-bromoindirubin 3'-oxime (BIO), a specific GSK-3 $\beta$ inhibitor, could promote N2A cells-derived neurons to become tumor-like neuroblasts according to morphological observation and marker staining. Consistently, we showed that constitutive activated $\beta$-catenin had the similar function. Moreover, the direct target genes of Wnt/ $\beta$-catenin signaling including $c-M y c$ and CyclinD1, as well as Id2 and Id3 were found to be greatly up-regulated which suggested the possible role of these genes during this cellular plasticity change process. In human neuroblastoma specimens, we found that the amount of activated $\beta$-catenin in nucleus was up-regulated significantly in pace with clinical neuroblastoma risk. These results suggest the important role of $\mathrm{Wnt} / \beta$-catenin signaling in modulation of cellular plasticity and possible functions in neuroblastoma origination.

\section{Materials and methods}

\section{Human tissue acquisition}

This study, approved by the Research Ethics Board of the Third Affiliated Hospital of Soochow University, was conducted on 12 patients undergoing surgery between 1990 and 2000, including 3 stroma-poor neuroblastomas, 5 stroma-rich neuroblastomas and 4 mixed histology with stroma-poor and stroma-rich regions. The tissues were embedded in paraffin for pathology diagnosis after surgery and were identified by Department of Pathology of Third Affiliated Hospital of Soochow University. Written informed consent was obtained from all patients or their representatives.

\section{Cell culture and cell counting}

Mouse N2A neuroblastoma cells were purchased from ATCC. N2A cells were cultured in Dulbecco's modified Eagle's medium (DMEM), containing 10\% FBS (Invitrogen, Carlsbad, CA) and penicillin/streptomycin $(100 \mathrm{U} / \mathrm{ml}$ and $100 \mathrm{mg} / \mathrm{ml}$, respectively) at $37{ }^{\circ} \mathrm{C}$ and $5 \% \mathrm{CO}_{2}$.

For the induction of neuronal differentiation, N2A cells were plated at a density of 100 cells $/ \mathrm{mm}^{2}$. After overnight incubation, cells were cultured in N2B27 medium for 96 hours. The medium was refreshed every 24 hours. Cells having one or more neurites of a length more than twice the diameter of the cell body were defined as post-mitotic neurons [12]. To quantify cell differentiation, eight randomly chosen fields from duplicate wells were photographed at each time point, and at least 800 cells were counted. Average percentage of differentiated cells was calculated from two to three independent experiments. BIO used in experiments was bought from Calbiochem (cat. no. 361550-1MG), and DKK1 protein was from R\&D company (cat. no.1765-DK-010).

\section{Cell transfection}

N2A cells were cultured in N2B27 medium for 96 hours and then transfected with pCAGGS-IRES-GFP, pCAGGS-S33Y- $\beta$-catenin-IRES-GFP plasmid, ctrl siRNA (target sequence: $5^{\prime}$ dTdTCdTCCGAACGdTGdTCACGdTdTdT3') or $\beta$-catenin-specific siRNA (target sequence: $5^{\prime}$ ACCATGCAGAATACAAATGATdTdT3') by lipofectmina 2000 (Invitrogen, Carlsbad, CA, USA) according to the manufacturer's instructions. Cells were harvested at 24 hours post transfection and prepared for immunofluorescence.

\section{Immunofluorescence}

Cells were fixed with $4 \%$ paraformaldehyde in PBS, and permeabilized with Triton X-100/Tris-buffered saline. For tissues, the paraffin was removed from human tissue sections with a standard procedure. Heat-induced antigen retrieval procedure was conducted at $92^{\circ} \mathrm{C}$ for 20 minutes with $0.01 \mathrm{M}$ citrate buffer ( $\mathrm{PH}$ 6.0). Then the following antibodies were used: Monoclonal, anti-Tuj1 (1:500, Covance), anti-Nestin (1:200, BD Pharmingen), anti- $\beta$-catenin (1:500, BD Pharmingen). Secondary antibody: anti-mouse-Cy3 (1:500, Jackson ImmunoResearch Laboratories) was used to visualize immunostaining. The images were taken with Olympus microscopy or Leica confocal microscopy SP2. Quantification of percentage of cells immunoreactive for Tuj1 (neuronal class III $\beta$-Tubulin, a neural stem cell marker) or Nestin antigens was determined by capturing images random fields. DAPI staining nuclei and cells positive for the Tuj1, Nestin were counted.

\section{Western blot}

For cell samples, plasmids (GFP or S33Y- $\beta$-catenin) or siRNAs (ctrl siRNA or $\beta$-catenin siRNA)-transfected or BIO-treated N2A cells were subjected for cytosol and nucleus protein analysis. The detailed methods were described previously [13]. For tissue samples, cytosol and nucleus proteins of each sample were subjected to Western blot analysis with the similar method. The cytosol or nucleus protein levels were calculated with software Image J, and 
the relative expression levels of $\beta$-catenin proteins in cytosol or nucleus were analyzed. Antibodies including $\beta$-catenin (BD Biosciences), H3-histone (Sig$\mathrm{ma})$ and $\beta$-actin (Cell Signaling) were used.

\section{Luciferase assay}

After N2A cell differentiation in N2B27 medium for 96 hours, TopFlash or FopFlash plasmid together with prl-tk plasmid were transfected into cells, accompanying with DMSO, BIO or $10 \%$ FBS treatment. The cells were harvested for luciferase assay at 24 hours after transfection with previously reported method [14].

\section{Cell cycle analysis}

After differentiated for 96 hours, N2A cells were treated with BIO for 24 hours. The cells were collected and washed with PBS, and then resuspended in $1 \mathrm{ml}$ DNA staining solution $(20 \mathrm{mg} / \mathrm{ml}$ of Propidium Iodide (PI) and $100 \mathrm{mg} / \mathrm{ml}$ of RnaseA in PBS) for $30 \mathrm{~min}$ on ice. DNA content was analyzed by FACS (Becton Dickinson, USA). The resulting DNA histograms were quantified using the Cell Quest Pro software.

\section{Real-time PCR}

Total mRNA was extracted from cells using Trizol reagent (Invitrogen, Carlsbad, CA, USA) according to the manufacturer's instructions. The reverse transcription was conducted with $2 \mu \mathrm{g}$ of total RNA using AMV reverse transcriptase (TaKaRa, Tokyo, Japan). Real-time PCR was performed using an Applied Biosystems 7500 Sequence Detection system (Applied Biosystems, Foster city, CA, USA) by standardized protocol. All reactions were run in triplicate and GAPDH was selected as an internal control. The relative amount of each mRNA to internal control GAPDH was calculated by using the equation $2^{-}{ }^{-} \mathrm{CT}$, in which $\triangle C_{T}=C_{T \text { mRNA }}-C_{T \text { GAPDH. }}$. The primers for real-time PCR are available upon request.

\section{Statistics}

Each experiment was repeated at least three times, and similar results were obtained. Data were presented as mean \pm s.d. Comparisons were performed using student's t-test. Differences were considered statistically significant at * $p<0.05$.

\section{Results and Discussion}

N2A cells were typical model for neuronal differentiation in vitro [15]. In the induction medium N2B27, N2A cells could differentiate into neurons with neurites (Fig. 1Aa-b). In our study, we found that the outgrowth of neurites and the typical neuronal phenotype of neurons that differentiated from N2A cells disappeared after re-feeding with 10\% FBS (Fig. $1 \mathrm{Ac})$, suggesting that some factors in the serum might cause the change of the cellular plasticity of N2A cells-derived neurons.

Considering the close relationship between $W n t / \beta$-catenin signaling and neural development, we wondered whether Wnt/ $\beta$-catenin pathway was involved in this process. Indeed, we found that DKK1 protein as an inhibitor of Wnt/ $\beta$-catenin pathway could block the FBS-induced change of the cellular plasticity of N2A cell-derived neurons (Fig. 1B). Furthermore, we found that $\mathrm{Wnt} / \beta$-catenin signaling was activated by adding $10 \%$ FBS as the luciferase activity of TopFlash was increased (Supplementary Material: Fig. S1), suggesting a potential role of Wnt pathway in this process. Therefore, we used BIO, a glycogen synthase kinase- $3 \beta$ (GSK3 $\beta$ ) inhibitor, which could stabilize $\beta$-catenin protein by preventing its degradation, to further examine the effects of activating Wnt $/ \beta$-catenin pathway. The results showed that BIO could significantly stimulate $\mathrm{Wnt} / \beta$-catenin pathway activity as indicated by the luciferase activity and protein levels of $\beta$-catenin in nuclei (Supplementary Material: Fig. S1 and Supplementary Material: Fig. S2 (lanes 1 and 2)). With different doses of BIO $(2 \mu \mathrm{M}$, $5 \mu \mathrm{M}$ and $10 \mu \mathrm{M})$, we found that neuronal phenotypes of N2A cells-derived neurons were gradually changed as doses of BIO increased (Fig. 1C). We also noticed that some cells presented cell death-like phenotype as nucleus was broken down when high doses of BIO $(5 \mu \mathrm{M}$ and $10 \mu \mathrm{M})$ were added (Fig. 1Cc-d). Therefore, we used $2 \mu \mathrm{M}$ BIO in our following experiments. These results showed that the N2A cell-derived neuron cell fate change was possibly dependent on BIO activation.

In order to confirm whether this phenotype change was caused by the activation of Wnt/ $\beta$-catenin pathway, constitutively activated $\beta$-catenin (S33Y- $\beta$-catenin) was transfected into neurons that induced from N2A cells to activate $W n t / \beta$-catenin signaling. The results showed that the protein levels of $\beta$-catenin were increased both in cytosol and nuclei after S33Y- $\beta$-catenin transfection (lanes 3 and 4 in Supplementary Material: Fig. S2), as a result, neurites and the neuronal phenotypes of S33Y- $\beta$-catenin highly expressed cells were significantly disappeared compared with the phenotypes of GFP-transfected cells (Fig. 2A). Quantitative assessment of neuron-like cells and neuroblast-like cells by cell morphology showed that S33Y- $\beta$-catenin significantly decreased the ratio of neuron-like cells from $46 \%$ to $18 \%$, and increased the ratio of neuroblast-like cells from $54 \%$ to $82 \%$ (Fig. 2B). To analyze whether endogenous 
$\beta$-catenin is responsible for BIO-induced effects, we employed $\beta$-catenin siRNA and demonstrated its efficiency (lanes 5 and 6 in Supplementary Material: Fig. S2). The results showed that the effects of BIO which promoted N2A cell-derived neurons to become neuroblastoma-like cells were significantly decreased when the expression of $\beta$-catenin was silenced (Figs.
2C and 2D), which strongly suggest that endogenous $\beta$-catenin mediated the role of BIO. Together with the above findings, these results suggest that activation of Wnt/ $\beta$-catenin pathway induced by BIO or constitutive activated $\beta$-catenin is responsible for $\mathrm{N} 2 \mathrm{~A}$ cell-derived neuron cell fate change.
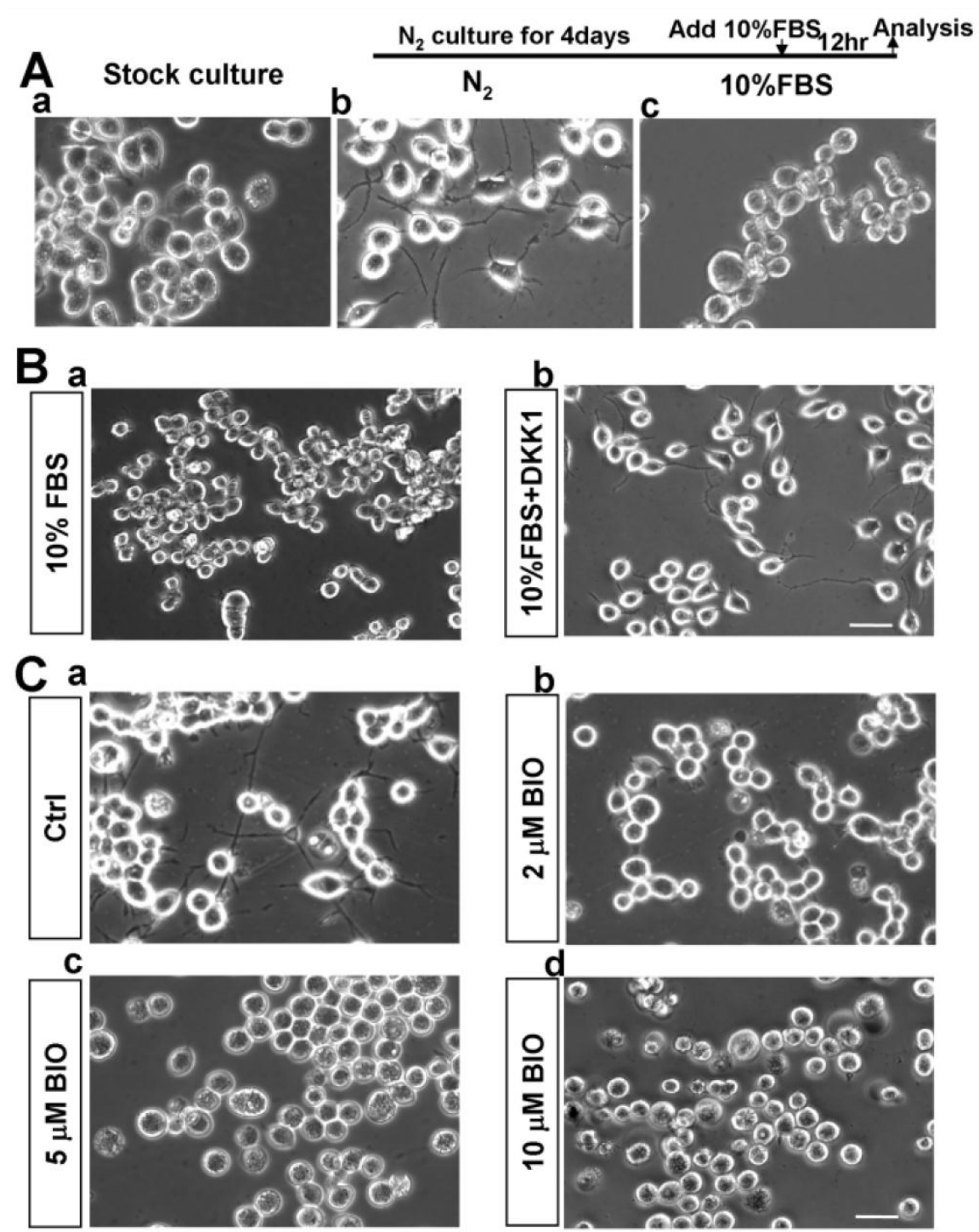

Fig. I BIO promotes N2A cell-derived neuron morphological changes. (A) N2A cells were induced to differentiate into neurons as previously described (b). Four days later, N2B27 medium was replaced by 10\% FBS (c). The induced neurite outgrowth was shown by brightfield images. Sclar bar $=200 \mu \mathrm{m}$. (B) After induction of N2A cells for 4 days, the cells were treated with $10 \%$ FBS (a) or $10 \%$

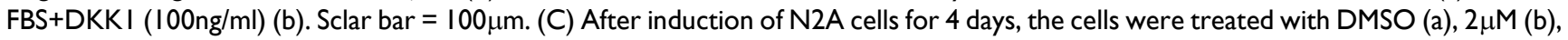
$5 \mu \mathrm{M}$ (c) or $10 \mu \mathrm{M}$ (d) BIO for 12 hours, respectively. Sclar bar $=100 \mu \mathrm{m}$ 

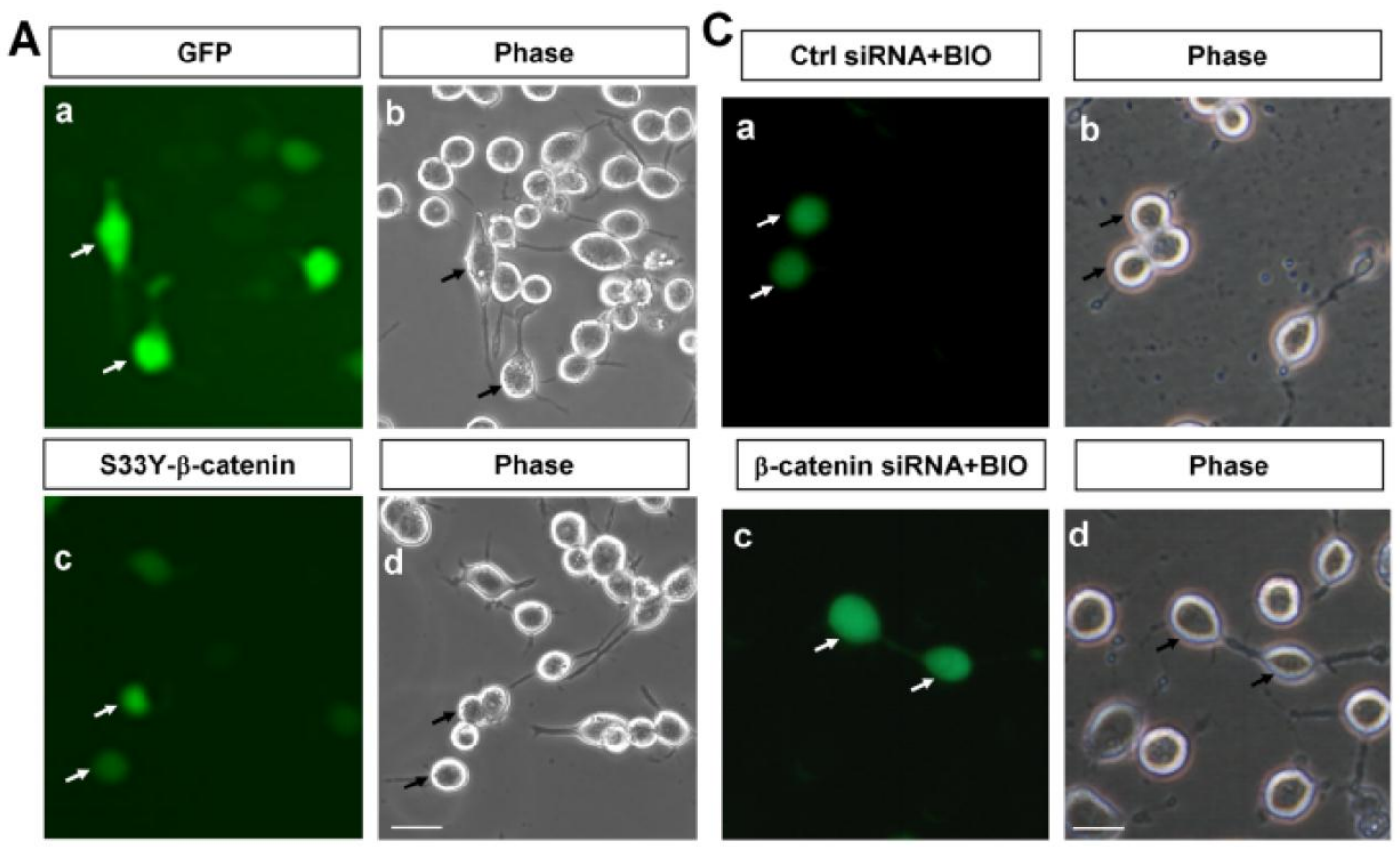

B
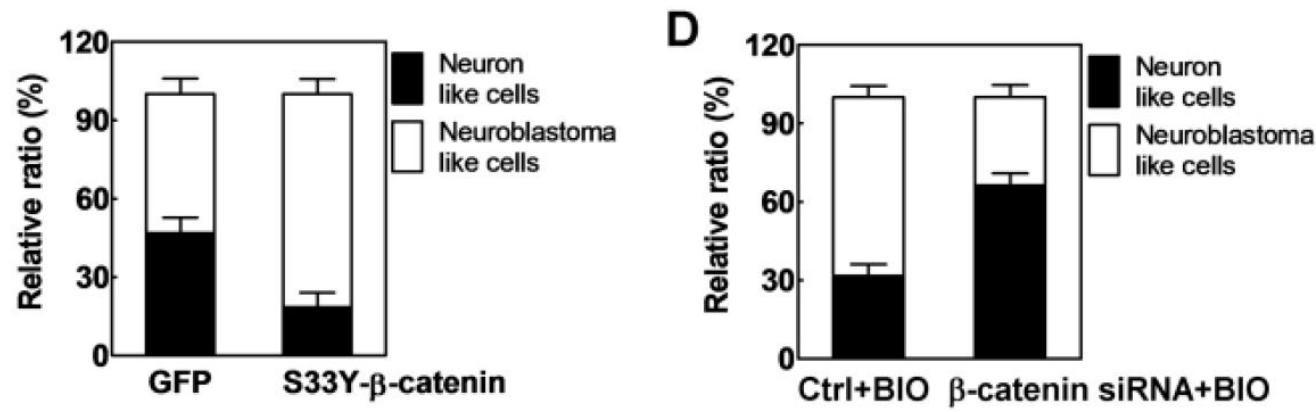

Fig. 2 Constitutive activated $\beta$-catenin modulates N2A cell-derived neuron cell fate. (A) After induction of N2A cells for 4 days, the morphology of GFP transfected cells (a, green) and S33Y- $\beta$-catenin transfected cells (c, green) were indicated by white arrow in corresponding immunofluorescence images and phase images. Sclar bar $=100 \mu \mathrm{m}$. (B) Quantitative assessment of neuron-like cells and neuroblastoma-like cells by cell morphology in GFP transfected cells and S33Y- $\beta$-catenin transfected cells. (C) After induction of N2A cells for 4 days, the morphology of GFP+Ctrl siRNA -transfected cells (a, green) and GFP+ $\beta$-catenin siRNA-transfected cells (c, green) were indicated by white arrow in corresponding immunofluorescence images and phase images. Sclar bar $=100 \mu \mathrm{m}$. (D) Quantitative assessment of neuron-like cells and neuroblastoma-like cells by cell morphology in GFP+Ctrl siRNA and GFP+ $\beta$-catenin siRNA-transfected cells.

To further confirm that N2A cells-derived neurons were changed as tumor-like neuroblasts after activation of Wnt/ $\beta$-catenin pathway by $\mathrm{BIO}$, we checked the expression of neuroblast marker Nestin and neuron marker Tuj1. The results showed that most cells were Nestin negative and Tuj1 positive neurons after induction of N2A cells in differentiation medium (Fig. 3A). After adding $2 \mu \mathrm{M}$ BIO, the number of Nestin positive neuroblasts was significantly increased, while the number of Tuj1 positive neurons was sharply reduced (Fig. 3A). Statistical analysis showed that Nestin positive NSCs raised from 19.5\% to $71.4 \%$, while Tuj 1 positive neurons decreased from $62.3 \%$ to $25.4 \%$ (Fig. $3 \mathrm{~B}$ ), which suggested that BIO could promote neurons into tumor-like neuroblasts. Real-time PCR was also used to test the expression changes of neuroblast markers such as Sox 2 and Nestin during this process. We found that the expression of Sox 2 and Nestin were significantly up-regulated for about 30 and 10 folds, respectively (Fig. 3C). We also examined the number of cells that reentered into cell cycle and found that the number of cells in cell cycle was significantly increased with BIO stimulation (Supplementary Material: Fig. S3). These data further supported the observation that neurons were changed into neuroblasts by BIO-activated Wnt/ $\beta$-catenin pathway. In previous studies, the Wnt signaling pathway has been demonstrated to participate in 
many normal and abnormal biological processes [16]. During neural development, Wnt/ $\beta$-catenin signaling pathway takes part in neural crest formation [17-18], neuronal differentiation as well as neurite outgrowth during cultured early-born hippocampus neuron differentiation [19-20]. In this study, we first promoted $\mathrm{N} 2 \mathrm{~A}$ cells to differentiate into neurons (differentiation for 4 days in serum-free medium), then activated $W n t / \beta$-catenin signaling in these N2A cell-derived neurons and found that activated Wnt/ $\beta$-catenin signaling forced these N2A cell-differentiated neurons to become tumor-like neuroblasts (Figs. 1-3). Combining these results with previous reports [19-20], we suggest that $\mathrm{Wnt} / \beta$-catenin signaling have distinct functions in different processes and different physiological conditions of neuronal differentiating. Here, our results reveal a novel function of Wnt/ $\beta$-catenin pathway in N2A cell-derived neuron cell fate modulation and imply the relationship of $\beta$-catenin-mediated activation and neuroblastoma formation.
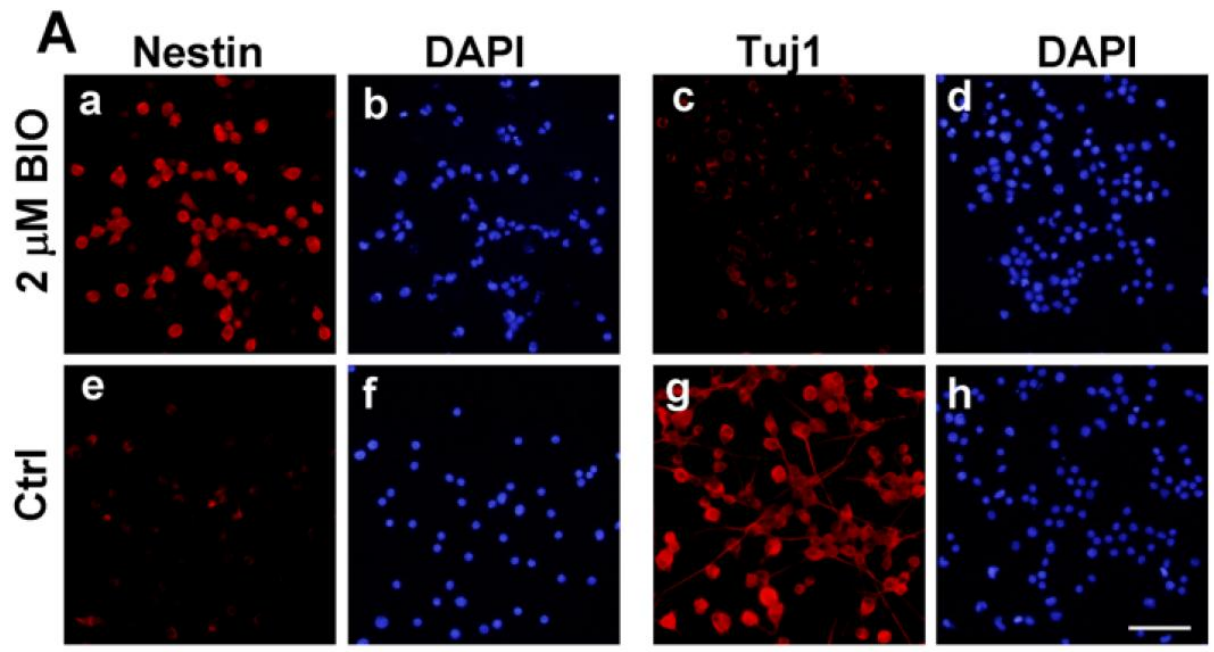

\section{B}

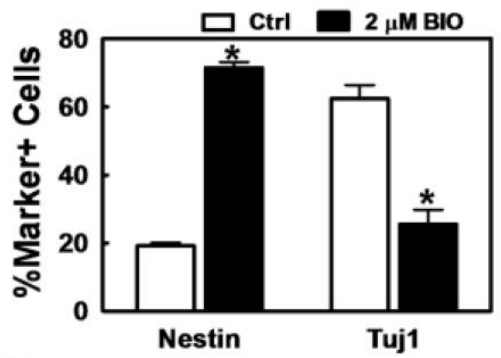

D
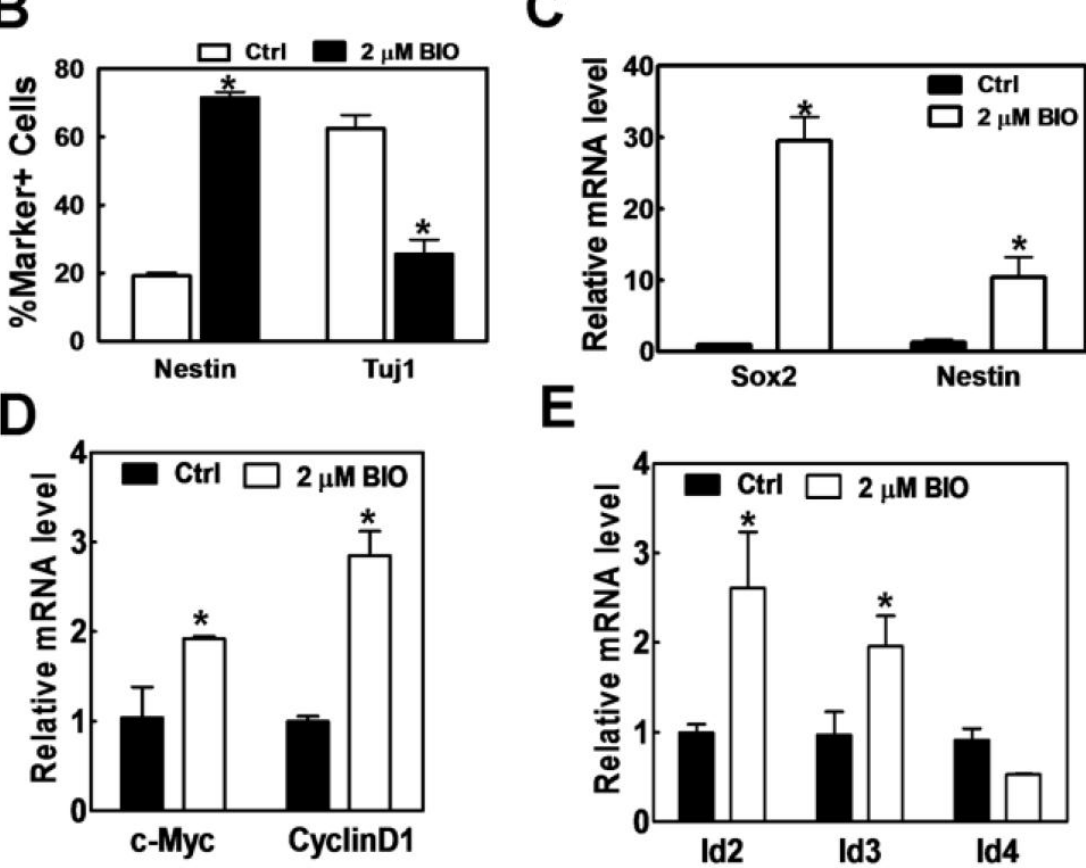

Fig.3 BIO promotes N2A cell-derived neurons back into tumor-like neuroblasts and up-regulates Id genes during this process. (A) Immunofluorescent staining of $\mathrm{N} 2 \mathrm{~A}$ cells and $2 \mu \mathrm{M}$ BIO treated N2A cells with anti-Nestin antibody (a and e, red), anti-Tuj I antibody (c and g, red) and DAPI (b, d, f and h, blue). Scale bar $=200 \mu \mathrm{m}$. (B) Quantitative assessment of Nestin positive and Tujl positive cells in control and BIO-treated N2A cells. (C) Relative mRNA expression level of NSC marker Sox 2 and Nestin in control and BIO-treated N2A cells. (D) Relative mRNA expression level of $c-M y c$ and CyclinDI in control and BIO-treated N2A cells. (E) Relative mRNA expression level of Ids in control and BIO-treated N2A cells. 


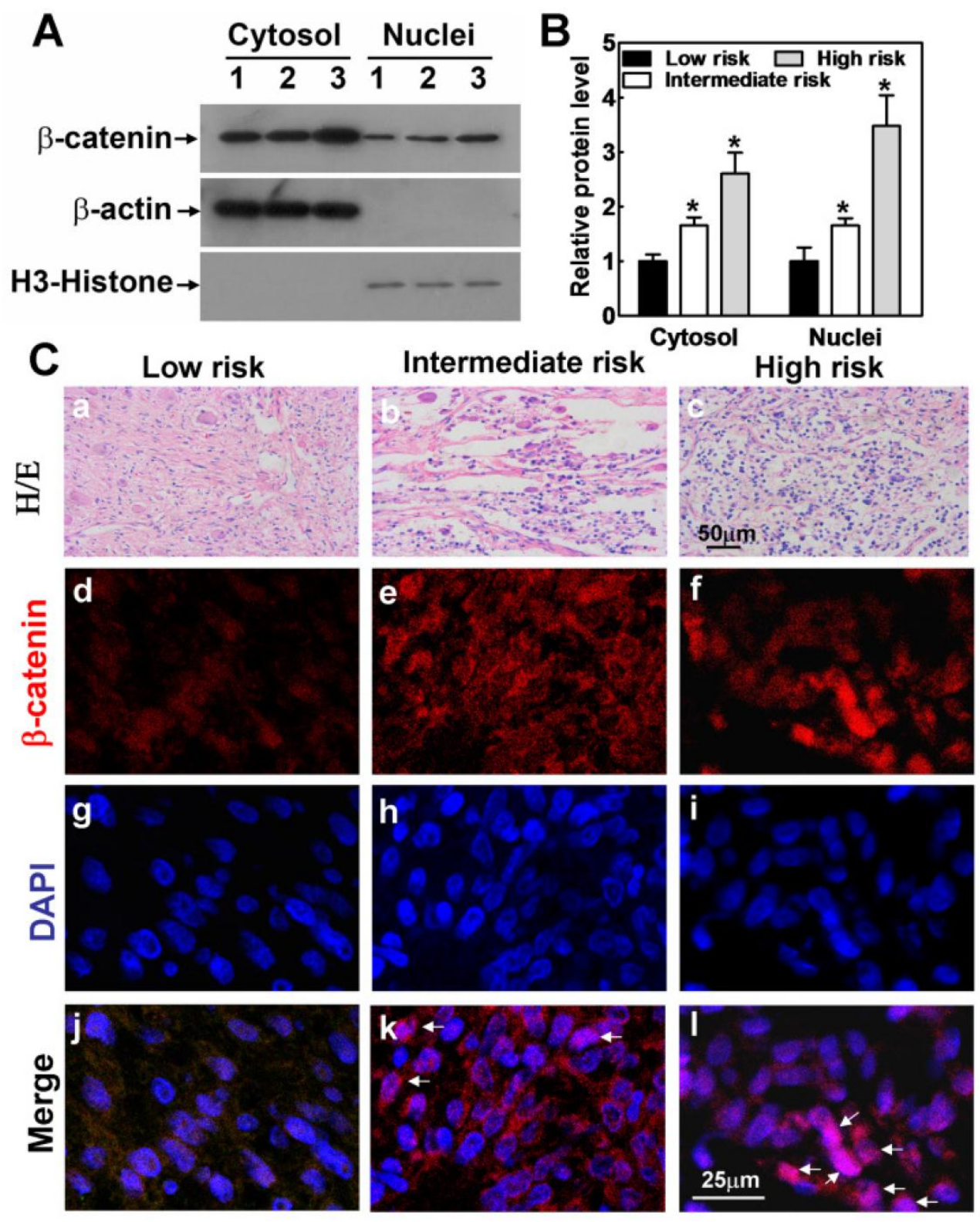

Fig.4 Activated $\beta$-catenin expression in clinical variants of human neuroblastoma. (A) Representative data of tissue sample Western blot. The expression of $\beta$-catenin protein in cytosol and nuclei was indicated. $\beta$-actin protein was used as a positive control for cytosol and negative control for nuclei, and $\mathrm{H} 3$-histone was used as a positive control for nuclei and negative control for cytosol. The symbols (I, 2, 3 ) indicated low, intermediate and high risk neuroblastoma tissue samples, respectively. (B) Quantitative assessment of the relative $\beta$-catenin protein levels of all tissue samples with Image J. The relative amount of $\beta$-catenin protein in cytosol and nuclei was normalized with $\beta$-actin and $\mathrm{H} 3$-histone, respectively. (C) (a-c) 3 stroma-poor deteriorated neuroblastomas with a favorable clinical outcome (low risk, a), 4 mixed histology with stroma-poor and stroma-rich regions (intermediate risk, b) and 5 stroma-rich neuroblastomas with poor outcome (high risk, c) were checked for their nucleus $\beta$-catenin levels ( $d$ for low risk, e for intermediate risk and $f$ for high risk). (d-l) The level of $\beta$-catenin in nucleus was higher in high risk neuroblastomas than in intermediate risk neuroblastomas and low risk neuroblastomas. Scale bar $=50 \mu \mathrm{m}$ for a-c, $=25 \mu \mathrm{m}$ for $\mathrm{d}-\mathrm{l}$.

To explore the molecular mechanisms, we examined the expression pattern of the downstream targets of Wnt/ $\beta$-catenin pathway, which may be involved in modulation of cellular plasticity. We first checked the direct target genes of Wnt/ $\beta$-catenin pathway such as $c-M y c$ and CyclinD1 and found that both genes are up-regulated upon Wnt signaling activation (Fig. 3D). In addition, we also observed that Id family genes, including Id2, Id3 and Id4 had a significant change during this process. $I d 2$ and $I d 3$ were significantly up-regulated (Fig. 3E). c-Myc and CyclinD1 proteins are important for cancer cell prolifer- 
ation [21] while Id proteins are involved in initiation and progression processes of many human tumors including endometrial carcinoma, melanoma, pancreatic carcinoma, head and neck cancers, and medullary thyroid carcinoma [22]. Studies found that Id proteins could transfer the phenotypic traits of embryonic stem cells to cancer cells [23-25], and mediate tumor reinitiation during tumor metastasis [26]. Our results showed that $c-M y c$ and $C y c l i n D 1$, as well as $I d 2$ and Id3 were significantly up-regulated by Wnt/ $\beta$-catenin signaling when N2A cell-derived neurons were forced to become tumor-like neuroblasts (Figs. 3D and 3E), suggesting that Id genes may mediate the function of $\mathrm{Wnt} / \beta$-catenin signaling in neuroblastoma initiation, and $c-M y c$ and $C y c l i n D 1$ play crucial role in neuroblastoma proliferation.

Considering $\beta$-catenin could promote N2A cell-derived neurons to become tumor-like neuroblasts, it is reasoned to propose that the activity of $\mathrm{Wnt} / \beta$-catenin pathway may be associated with human neuroblastoma progression in situ. We examined the $\beta$-catenin protein levels in nucleus of 12 human neuroblastomas, 5 stroma-rich neuroblastomas with poor outcome (high risk), 4 mixed histology with stroma-poor and stroma-rich regions (intermediate risk), and 3 stroma-poor neuroblastomas with a favorable clinical outcome (low risk). We analyzed all the tissue samples we have, and presented the representative Western blot data to show that the levels of $\beta$-catenin protein in cytosol and nucleus were gradually up-regulated as the risk of neuroblastomas increased (Fig. 4A). Statistical analysis of all the Western blot data further supported our notion that activated $\beta$-catenin protein levels in nucleus were correlated with neuroblastoma clinical outcome (Fig. 4B). Consistently, the immunofluorescence experiment also showed that the level of $\beta$-catenin in nucleus was evidently up-regulated in both intermediate risk and high risk neuroblastomas compared with low risk neuroblastomas (Fig. 4Cd-1). Thus, the data suggest that the stronger the $\beta$-catenin expressed in nucleus, the higher risk of neuroblastoma, and the poorer the prognosis is. Taken the above results together, we could refer that $\beta$-catenin in nucleus is one of the key factors in determining neuroblastoma carcinogenesis. Consistent with our results, Wnt/ $\beta$-catenin signaling inhibitors such as DDK1 are found to be effective methods to treat neuroblastoma in vitro [27]. Therefore, these studies suggest a potential key role of $\beta$-catenin in human neuroblastoma progression and prognosis which may further provide us a new insight into the cure of neuroblastoma.

\section{Conclusion}

In this study, we revealed that BIO (a specific GSK-3 $\beta$ inhibitor) or activated $\beta$-catenin could promote N2A cell-derived neurons to become tumor-like neuroblasts which implicated the potential relationship between the Wnt/ $\beta$-catenin signaling and neuroblastoma formation. Furthermore, we found that the amount of $\beta$-catenin in nucleus was accumulated in human neuroblastoma specimens and positively correlated with clinical risk of neuroblastoma. Taken together, these results may provide us a new sight into the initiation, progression and cure of neuroblastoma.

\section{Supplementary Material}

Fig.S1: FBS and BIO stimulate Wnt/b-catenin activities. Fig.S2: The expression levels of b-catenin protein in cytosol and nuclei. Fig.S3: BIO promotes N2A cell-derived neurons to reenter cell cycle. http://www.biolsci.org/v08p0289s1.pdf

\section{Acknowledgments}

We would like to thank Prof. Xi Chen from Nanjing University for helpful discussions on the manuscript. This work was supported in part by NSFC 31071046, CSDP (CS20092015), CHB (ZD200903), CSDP (CS20102010), CHB (ZD201007).

\section{Conflict of Interests}

The authors have declared that no conflict of interest exists.

\section{References}

1. Hagg T. Endogenous regulators of adult CNS neurogenesis. Curr Pharm Des. 2007; 13(18): 1829-1840.

2. Nakagawara A, Ohira M. Comprehensive genomics linking between neural development and cancer: neuroblastoma as a model. Cancer Lett. 2004; 204(2): 213-224.

3. Pahlman S, Stockhausen MT, Fredlund E, et al. Notch signaling in neuroblastoma. Semin Cancer Biol. 2004; 14(5): 365-373.

4. Mazieres J, He B, You L, et al. Wnt signaling in lung cancer. Cancer Lett. 2005; 222(1): 1-10.

5. Kleber M, Lee HY, Wurdak $\mathrm{H}$, et al. Neural crest stem cell maintenance by combinatorial Wnt and BMP signaling. J Cell Biol. 2005; 169(2): 309-320.

6. Eckfeldt CE, Mendenhall EM, Verfaillie EM. The molecular repertoire of the 'almighty' stem cell. Nat Rev Mol Cell Biol. 2005; 6(9): 726-737.

7. Mikkers H, Frisen J. Deconstructing stemness. EMBO J. 2005; 24(15): 2715-2719.

8. Hirsch C, Campano LM, Wohrle S, et al. Canonical Wnt signaling transiently stimulates proliferation and enhances neurogenesis in neonatal neural progenitor cultures. Exp Cell Res. 2007; 313(3): 572-587.

9. Muroyama $\mathrm{Y}, \mathrm{Kondoh} \mathrm{H}, \mathrm{Takada} \mathrm{S}$. Wnt proteins promote neuronal differentiation in neural stem cell culture. Biochem Biophys Res Commun. 2004; 313(4): 915-921. 
10. Marson A, Foreman R, Chevalier B, et al. Wnt signaling promotes reprogramming of somatic cells to pluripotency. Cell Stem Cell. 2008; 3(2): 132-135.

11. Lluis F, Pedone E, Pepe S, et al. The Wnt/ beta-catenin signaling pathway tips the balance between apoptosis and reprogramming of cell-fusion hybrids. Stem Cells. 2010; 28(11): 1940-1949.

12. Cowley S, Paterson $\mathrm{H}, \mathrm{Kemp} P$, et al. Activation of MAP kinase kinase is necessary and sufficient for PC12 differentiation and for transformation of NIH 3 T3 cells. Cell. 1994; 77(6): 841-852.

13. Jian $H$, Shen $X$, Liu I, et al. Smad3-dependent nuclear translocation of beta-catenin is required for TGF-beta1-induced proliferation of bone marrow-derived adult human mesenchymal stem cells. Genes Dev. 2006; 20(6): 666-74.

14. Xie Z, Chen Y, Li Z, et al. Smad6 promotes neuronal differentiation in the intermediate zone of the dorsal neural tube by inhibition of the Wnt/beta-catenin pathway. Proc Natl Acad Sci USA. 2011; 108(29): 12119-24.

15. Hargreaves AJ, Fowler MJ, Sachana M, et al. Inhibition of neurite outgrowth in differentiating mouse N2a neuroblastoma cells by phenyl saligenin phosphate: effects on MAP kinase (ERK 1/2) activation, neurofilament heavy chain phosphorylation and neuropathy target esterase activity. Biochem Pharmacol. 2006; 71(8): 1240-1247.

16. Cadigan KM, Nusse R. Wnt signaling: a common theme in animal development. Genes Dev. 1997; 11(24): 3286-3305.

17. Garcia-Castro MI, Marcelle C, Bronner-Fraser M. Ectodermal Wnt function as a neural crest inducer. Science. 2002; 297(5582): 848-851.

18. Wu J, Saint-Jeannet JP, Klein PS. Wnt-frizzled signaling in neural crest formation. Trends Neurosci. 2003; 26(1): 40-45.

19. David MD, Canti C, Herreros J. Wnt-3a and Wnt-3 differently stimulate proliferation and neurogenesis of spinal neural precursors and promote neurite outgrowth by canonical signaling. J Neurosci Res. 2010; 88(14): 3011-3023.

20. Colombres M, Henriquez JP, Reig GF, et al. Heparin activates Wnt signaling for neuronal morphogenesis. J Cell Physiol. 2008; 216(3): 805-815.

21. Lee E, Muller W. Oncogenes and Tumor Suppressor Genes. Cold Spring Harb Perspect Biol. 2010; 2: a003236.

22. Ruzinova MB, Benezra R. Id proteins in development, cell cycle and cancer. Trends Cell Biol. 2003; 13(8): 410-418.

23. Norton JD. ID helix-loop-helix proteins in cell growth, differentiation and tumorigenesis. J Cell Sci. 2000; 113 ( Pt 22): 3897-3905.

24. Sikder H A, Devlin MK, Dunlap S, et al. Id proteins in cell growth and tumorigenesis. Cancer Cell. 2003; 3(6): 525-530.

25. Iavarone A, Lasorella A. ID proteins as targets in cancer and tools in neurobiology. Trends Mol Med. 2006; 12(12): 588-594.

26. Gupta GP, Perk J, Acharyya S, et al. ID genes mediate tumor reinitiation during breast cancer lung metastasis. Proc Natl Acad Sci U S A. 2007; 104(49): 19506-19511.

27. Koppen A, Ait-Aissa R, Hopman S, et al. Dickkopf-1 is down-regulated by MYCN and inhibits neuroblastoma cell proliferation. Cancer Lett. 2007; 256(2): 218-228. 